\title{
Activity of Phenylalanine Ammonia-Lyase (PAL) and Concentrations of Anthocyanins and Phenolics in Developing Strawberry Fruit
}

\author{
Guiwen W. Cheng and Patrick J. Breen \\ Department of Horticulture, Oregon State University, Corvallis, OR 97331 \\ . Additional index words. Fragaria $x$ ananassa, tannins, flavonoids, chlorophyll
}

\begin{abstract}
Studies on regulation of production of phenolics in strawberry (Fragaria $X$ ananassa Duch,) fruit were initiated by monitoring phenylalanine ammonia-lyase (PAL) activity and levels of anthocyanins, flavonoids, tannins, and other soluble phenols throughout fruit ontogeny in 'Tillikum'. PAL catalyzes the first step in the biosynthesis of phenylpropanoids, which are further modified into a wide variety of phenolic compounds. Peak in PAL activity (1 mol $\cdot \mathrm{s}^{-1}=1 \mathrm{~kat}$ ) of 90 pkat $\mathrm{mg}^{-1}$ protein was detected at 5 and 27 days after anthesis (DAA), when fruit was green and nearly ripe, respectively. PAL activity was only $\approx 10 \%$ of peak values in the white berry stage, when. fruit growth was most rapid. The second peak in PAL activity was followed by a rapid drop, to nearly zero in red-ripe fruit at 30 DAA. Total soluble phenols reached a maximum level soon after anthesis, just before the first peak in PAL activity, then declined to a low constant value well in advance of fruit ripening. Similar changes were observed in levels of tannins and flavonoids that, at anthesis, accounted for $44 \%$ and $51 \%$ of the soluble phenols, respectively. The concentration of anthocyanin was very low throughout most of fruit development, but beginning at 23 DAA it increased from $<0.03$ to $>0.53 \mathrm{mg} \cdot \mathrm{g}^{-1}$ fresh weight in 3 days. This accumulation paralleled the second rise in PAL activity. Accordingly, strawberry fruit have a developmental-dependent expression of PAL activity and accumulation of phenolic substances derived from the phenylpropanoid pathway.
\end{abstract}

Many distinctive developmental features of fleshy fruits, such as loss of astringency and appearance of characteristic color at ripening, are related to changes in the synthesis and accumulation of phenolic compounds. For example, astringency is a tannin effect and the color of ripe fruit often results from accumulated anthocyanins. Besides anthocyanins and condensed tannins (proanthocyanins), fruit also contain other flavonoids, as well as simple phenols such as phenolic acids and cinnamic acid derivatives (see review by Macheix et al., 1990). The type and amount of phenolic compounds in plant tissues depend on genotype and developmental stage (Hahlbrock and Griseback, 1-979). Tissues of fleshy fruits commonly have high levels of cinnamic acid derivatives, flavonoids, and tannins during early development, but accumulate anthocyanins only near maturity (Billet et al., 1978; Hyodo, 1971; Kataoka et al., 1983). Information on metabolism of phenolics is important not only because they influence the quality of fruit and related processed products, but also because of their roles in developmental physiology and defense mechanisms.

Understanding phenolic metabolism in plant cells and tissues requires knowledge of the biosynthetic reactions and their regulation. The majority of monophenolic compounds, most of which are phenolic acids and phenylpropanoids, are intermediates and derivatives of the shikimate and phenylpropanoid pathways. Flavonoids, including anthocyanins and condensed tannins, are derived from p-coumaric acid of the phenylpropanoid pathway. Another group of tannins, the hydrolyzable tannins, are ester derivatives of gallic acid that maybe synthesized via the shikimate or phenylpropanoid pathways (Ishikura et al., 1984). Conversion of L-phenylalanine to trans -cinnamic acid is the initial step of the phenylpropanoid pathway. This ammonia elimination reaction is catalyzed by L-phenylalanine ammonialyase (PAL) (EC 4.3.1.5), a key regulatory enzyme in the bio-

Received for publication 28 Dec. 1990. Oregon Agricultural Experiment Station Technical Paper no. 9476. The cost of publishing this paper was defrayed in part by the payment of page charges. Under postal regulations, this paper therefore must be hereby marked advertisement solely to indicate this fact. synthesis of phenolics (Jones, 1984). High PAL activity is associated with the accumulation of anthocyanins and other phenolic compounds in fruit tissues of several species (Billet et al., 1978; Blankenship and Unrath, 1988; Kataoka et al., 1983). Recent work of Given et al. (1988a) shows that accumulation of anthocyanins in ripening strawberry fruit requires high PAL activity. However, we found no reports of either the changes in total soluble phenolics, flavonoids, and tannins or their relationships to PAL activity throughout development of the strawberry fruit.

We monitored PAL activity and quantified changes in anthocyanins, tannins, flavonoids, and total soluble phenols in strawberry fruit from anthesis to ripeness. The aim of the work was to provide a basis for future studies on the biological mechanisms regulating the production of phenolics in developing strawberry fruit.

\section{Materials and Methods}

Certified nursery plants of 'Tillikum', a day-neutral strawberry that produces small to medium-sized fruit (Sjulin and Barritt, 1984), were grown in 3.8-liter pots in 1 soil : 1 coarse sand $: 1$ peat (by volume) in a greenhouse that was maintained at $30 /$ $18 \mathrm{C}$ (day/night) and where high-pressure sodium lamps (175 \pm $\left.25 \mu \mathrm{mol} \cdot \mathrm{m}^{-2} \cdot \mathrm{s}^{-1}\right)$ provided a $16-\mathrm{h}$ photoperiod. Flower buds were removed during the first 40 days after planting to encourage vegetative growth. Subsequently, flowers at anthesis were tagged and hand-pollinated to ensure normal fruit growth. Flower buds at the tertiary rank and below were removed to improve growth uniformity of secondary fruit, which were collected for analysis.

PAL extraction and assay. Prechilled fruit were ground with pestle and mortar placed on dry ice. The resulting tissue powder was well mixed with chilled acetone, placed in a freezer for 15 rein, and then centrifuged at $20,000 \times \mathrm{g}$ at $4 \mathrm{C}$ for $15 \mathrm{~min}$. The pellet was dried under vacuum and extracted at $4 \mathrm{C}$ by gentle stirring with $100 \mathrm{mM}$ sodium borate buffer $\left(5 \mathrm{ml} \cdot \mathrm{g}^{-1}\right.$ fresh weight),

Abbreviations: DAA, days after anthesis; PAL, phenylalanine ammonia-lyase. 
pH 8.8, containing $5 \mathrm{mM}$ B-mercaptoethanol, $2 \mathrm{mM}$ EDTA, and acid-washed polyvinylpolypyrrolidine at $10 \%$ the fresh weight. After $1 \mathrm{~h}$, the solution was filtered through one layer of nylon cloth and centrifuged as above.

PAL activity in the buffer supernatant was determined by the production of cinnamate during $1 \mathrm{~h}$ at $30 \mathrm{C}$, as measured by the absorbance change at $290 \mathrm{~nm}$ (Zucker, 1965). The assay mixture contained $15 \mu \mathrm{mol}$ L-phenylalanine, $30 \mathrm{mM}$ sodium borate buffer ( $\mathrm{pH} 8.8$ ), and 0.2 to $0.5 \mathrm{ml}$ buffer supernatant, depending on the PAL activity level, in a total volume of $3.0 \mathrm{ml}$. The substrate was added after $10 \mathrm{~min}$ of preincubation and the reactions stopped with $0.1 \mathrm{ml} 6 \mathrm{~N} \mathrm{HCl}$. Assays were performed in triplicate. Under the experimental conditions, the increase in absorbance at $290 \mathrm{~nm}$ was linear for up to $3 \mathrm{~h}$. The molar extinction coefficient of authentic trans-cinnamic acid in assay buffer plus HCI was determined to be 17,400. A unit of enzyme activity (kat) was defined as the amount of enzyme required for the formation of $1 \mathrm{~mol}$ of product in $1 \mathrm{sec}$ under the assay conditions.

Identification of reaction product. The reaction product was identified as cinnamic acid by using ${ }^{14} \mathrm{C}$ L-phenylalanine (ICN Radiochemical, Irvine, Calif.) and thin layer chromatography (TLC). One-dimensional ascending TLC was carried out on silica plates (G60 F254, EM Science, Gibbstown, N. J.) with three separate solvent systems-benzene : acetic acid $(4: 1, \mathrm{v} / \mathrm{v})$, benzene : methanol : acetic acid (45:8:4), and ethanol : ammonia : water $(80: 4: 16)$. Spots on the plates were detected under UV light. In all solvent systems, the $\mathrm{Rf}$ values of the ${ }^{14} \mathrm{C}$-reaction product were identical to those of authentic trans-cinnamic acid. Radioactivity of the reaction products was confirmed by scraping off the spot, extracting with ethyl ether, and measuring ${ }^{14} \mathrm{C}$ activity in a scintillation counter.

Protein. Protein was determined using the dye-binding assay of Bradford (1976) with bovine serum albumin as a standard.

Chlorophyll. Chlorophyll in the acetone supernatant was determined from absorbance at 645 and $663 \mathrm{~nm}$ according to Mackinney (1941).

Anthocyanin. Anthocyanin was estimated by a $\mathrm{pH}$ differential method. Absorbance was measured at $515 \mathrm{~nm}$, and $700 \mathrm{~nm}$ for eliminating interference from background turbidity, in buffers at $\mathrm{pH} 1.0$ and 4.5 , using $\mathrm{A}=\left(\mathrm{A}_{515}-\mathrm{A}_{700}\right)_{\mathrm{pH} 1 . \mathrm{o}}-\left(\mathrm{A}_{515}-\right.$ $\left.\mathrm{A}_{700}\right)_{\mathrm{H} 45}$, and ${ }^{\mathrm{a}}$ molar extinction coefficient of 22,400 (Pilando et al., 1985). Acetone extraction (i.e., acetone supernatant from the PAL extraction) was used. Preliminary experiments indicated that the recovery of anthocyanins with acetone extraction was equal to that with $0.1 \% \mathrm{HC} 1$ methanol. Results were expressed as milligrams of pelargonidin-3-glucoside per gram fresh weight.

Phenolics. Fruit was ground with pestle and mortar with $95 \%$ ethanol ( $2 \mathrm{ml} \cdot \mathrm{g}^{-1}$ fresh weight each). The pellet, after centrifugation, was again extracted with $95 \%$ ethanol and then once with $5 \%$ ethanol in the same procedure. Total soluble phenols in the combined supernatants were determined with Folin-Ciocalteu reagent by the method of Slinkard and Singleton (1977). Total soluble phenols were separated into three fractions according to the method of Peri and Pompei (1971). Tannins were precipitated by cinchonine and dissolved in ethanol-HCl. The supernatant was treated with formaldehyde; nonflavonoid phenolics (NF-phenolics) remained in solution and nontannin flavonoids (NT-flavonoids), determined by difference, precipitated. Results were expressed as milligrams gallic acid equivalent (GAE) per gram fresh weight.

\section{Results}

Fruit growth. Secondary fruit of 'Tillikum' grew slowly for $\approx 15$ DAA, then rapidly gained weight, approaching a maximum weight of $\approx 4.5 \mathrm{~g}$ by 25 DAA (Fig. 1). Fruit turned from green to white at $\approx 15 \mathrm{DAA}$, showed pink at $23 \mathrm{DAA}$, and was red-ripe at $30 \mathrm{DAA}$.

Chlorophyll. The concentration of chlorophyll in young fruit was $70 \mu \mathrm{g} \cdot \mathrm{g}^{-1}$ soon after anthesis but then rapidly dropped to $\approx 10 \mathrm{~g}^{-1}$ by $15 \mathrm{DAA}$, just before the rapid burst in fruit growth (Fig. 1). As shown by changes in the amount of chlorophyll per fruit, chlorophyll formation continued for up to 15 DAA (Fig. 1).

Phenolics. The concentration of total soluble phenols reached a maximum of $194 \mathrm{mg} \mathrm{GAE} / \mathrm{g}$ during fruit set, then rapidly decreased, falling to a minimum, stable value of $\approx 8 \mathrm{mg} \mathrm{GAE} /$ g by 19 DAA (Fig. 2). Even though the concentration of total soluble phenols continuously decreased, the amount steadily increased (per berry) (Fig. 2). Patterns of change in concentration

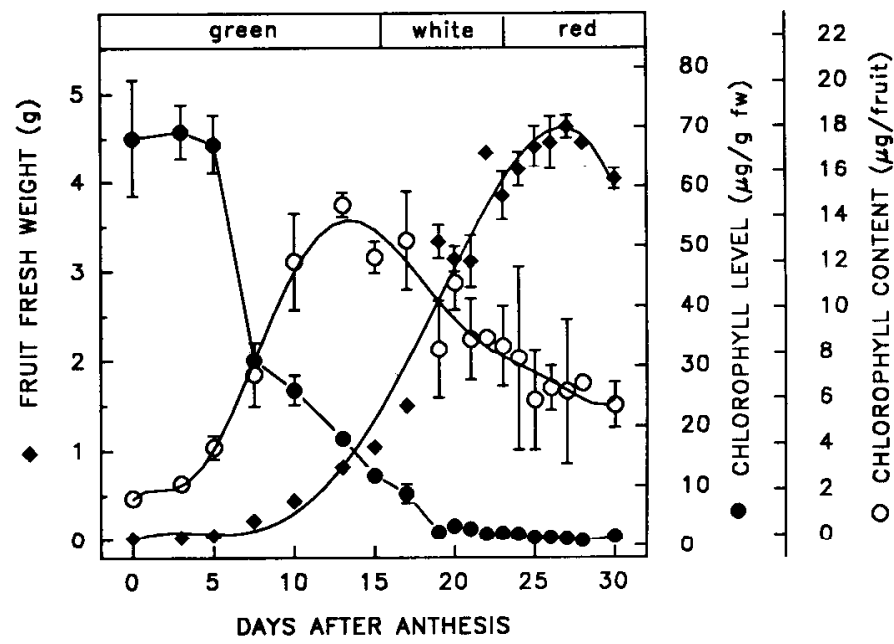

Fig. 1. Changes in color, fresh weight, and chlorophyll level and content in the developing secondary fruit of 'Tillikum' strawberry. Vertical bars represent \pm SE.

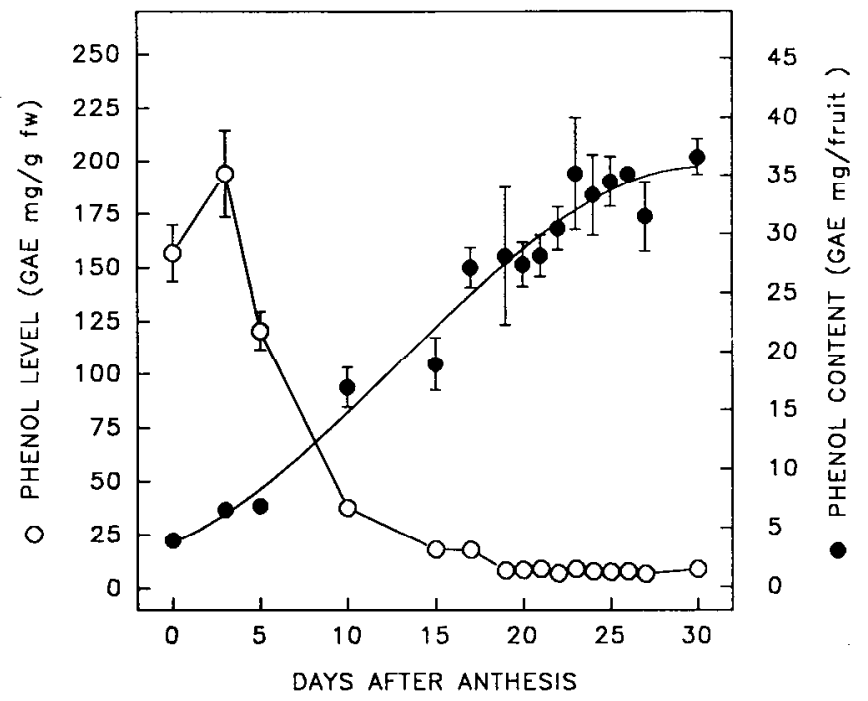

Fig. 2. Changes in level and content of total soluble phenols of the developing secondary fruit of 'Tillikum' strawberry. Vertical bars represent \pm SE. 
of both tannins and NT-flavonoids in fruit reflected that of total soluble phenols (Fig. 3). At their peaks, tannins and NT-flavonoids accounted for $44 \%$ and $51 \%$, respectively, of total phenols. The NF-phenolics never accounted for $>5 \%$ of the total. Most of the increase in total soluble phenol content on a fruit basis was due to increases in NT-flavonoids.

Anthocyanins. Anthocyanins were undetectable during the white berry stage (Fig. 4A). Low absorbance was recorded in young fruit, but this was likely an artifact due to interference from chlorophyll. Anthocyanins were not detected when phenolics from green fruit were separated by high performance liquid chromatography using the method of Spanos and Wrolstad (1990). Anthocyanins began to accumulate rapidly at 23 DAA, and the concentration increased within a week to a maximum of $\approx 0.65$ $\mathrm{mg} \cdot \mathrm{g}^{-1}$ in ripe fruit. Anthocyanin content per fruit showed a similar pattern.

$P A L$. Two peaks of PAL activity occurred during strawberry fruit development, each with a rate of $90 \mathrm{pkat} \cdot \mathrm{mg}^{-1}$ protein (Fig. 4B). The first peak occurred within a week after anthesis and was followed by a decline to $\approx 10 \mathrm{pkat} \cdot \mathrm{mg}^{-1}$ protein by 15 DAA, where it remained for 7 days. A sharp rise in activity began on 23 DAA, concomitant with the rise in anthocyanin accumulation, and reached the second peak 27 DAA. PAL activity then underwent a rapid decline and was nearly undetectable at 30 DAA. At 6 DAA, the total PAL activity in receptacle and achenes was equal, even though the fresh weight of receptacle tissue was four times that of the achenes (data not shown). For comparison, PAL activity was also plotted on per-gram fresh weight and per-fruit basis in Fig. 4C. Clearly, total PAL activity per fruit was much higher in ripening than in young fruit.

\section{Discussion}

Development of 'Tillikum' strawberry fruit can be visually separated into green, white, and red berry stages. These stages reflect changes in chlorophyll and anthocyanin levels as well as distinctive differences in growth and development, phenolic levels, and PAL activity.

The 15 days of the green berry stage accounted for about half of the total period of fruit development, although the fruit attained $<25 \%$ of its final fresh weight. While the fruit chloro-

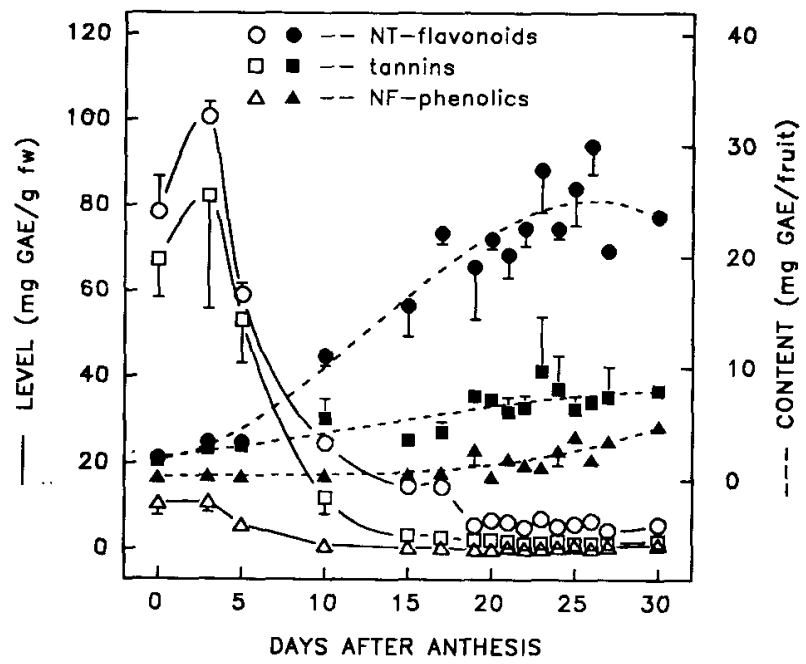

Fig. 3. Changes in level and content of tannins, nontannin (NT) flavonoids and nonflavonoid (NF) phenolics in the developing fruit of 'Tillikum' strawberry. Vertical bars represent $\pm \mathrm{SE}$.

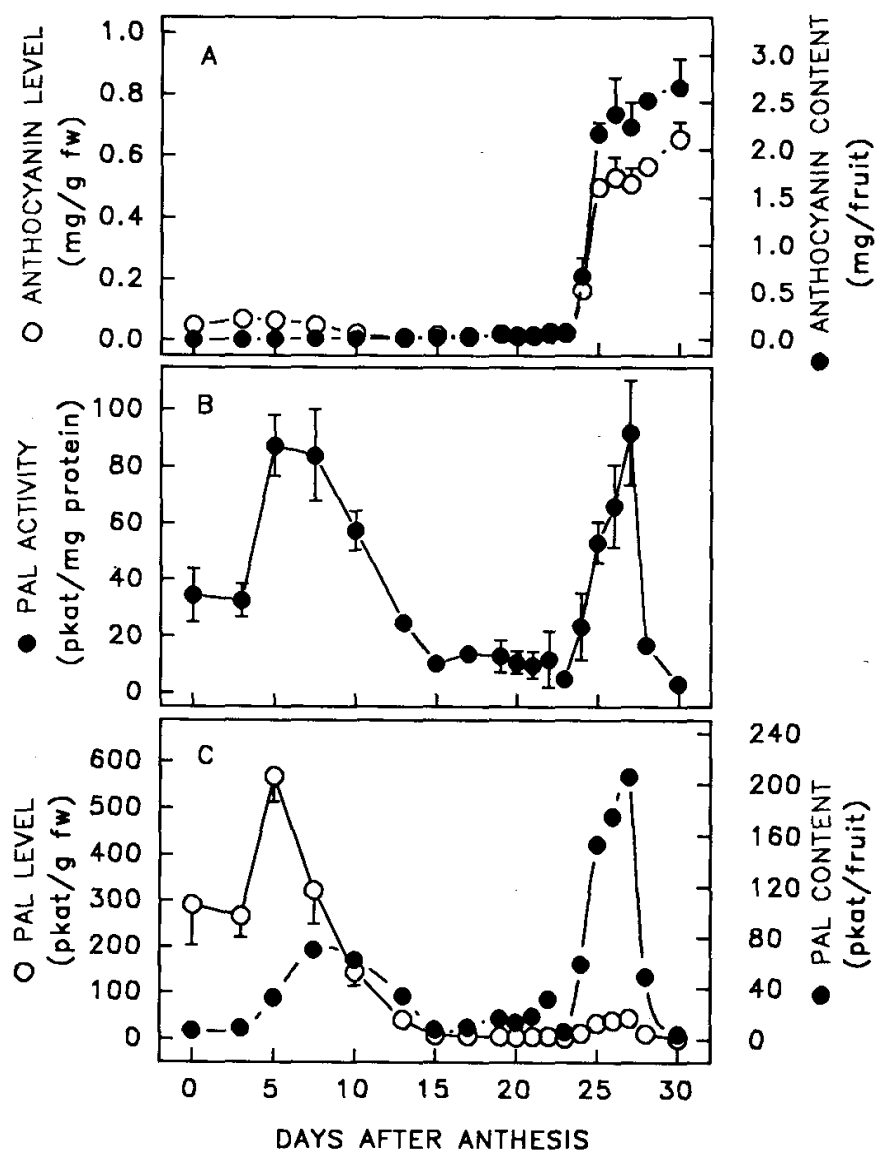

Fig. 4. Developmental changes in (A) anthocyanin accumulation and activity of PAL expressed on a (B) protein and (C) fresh weight and whole fruit basis in the secondary fruit of 'Tillikum' strawberry. Vertical bars represent \pm SE.

phyll content increased throughout the entire 15 -day period, the concentration decreased rapidly following anthesis. Similar changes occur in 'Red Gauntlet' strawberry fruit (Woodward, 1972).

The very high concentration of phenolics in young 'Tillikum' fruit is a common feature in strawberry cultivars (Spayd and Morris, 1981) and other fruit species (Billot et al., 1978; Hyodo and Asahara, 1973; Melin et al., 1977). When achenes and receptacle were separated before analysis, $58 \%$ of the total soluble phenols in strawberry fruit at anthesis was found in achenes (data not shown). Since the achenes contributed $56 \%$ of the total fruit fresh weight, the concentration of total soluble phenols was similar in achenes and receptacle early in fruit development. During this period, the total soluble phenols consisted essentially of equal portions of tannins and NT-flavonoids. An appreciable share of the tannin fraction was likely condensed tannins, since strawberry fruit reportedly contains substantial amounts of these substances (Jersch et al., 1989). Foo and Porter $(1980)$ found that condensed tannins account for $74 \%$ and $63 \%$ of the total tannins (condensed + hydrolyzable) isolated from unripe and ripe strawberry fruit. Changes in the concentration of total soluble phenols soon after anthesis were broadly associated with, but not parallel to, the first rise in PAL activity. Total soluble phenols reached a peak and began to decline a few days before the maximum in PAL activity was observed. A close parallel between PAL activity and phenol levels and a lag in the peak in phenols has been reported in other fruit species (Billot et al., 1978; Kataoka et al., 1983; Kubo et al., 1988; 
Melin et al., 1977). The apparent discrepancy between PAL activity and level of total soluble phenols in this study may have resulted from accumulation of phenols before anthesis, active biosynthesis of insoluble phenolic compounds (e.g., polymeric proanthocyanins, etc.), or difference in the sensitivity of the phenol assay to various phenolics. Difference between in vivo and in vitro PAL activity also may have contributed to the discrepancy since measurement of PAL can be extremely variable when activity is high, such as in young fruit of pear (Billet et al., 1978) and cherry (Melin et al., 1977), or during fruit maturation in strawberry (Aoki et al., 1970) and apple (Kubo et al., 1988).

Fruit growth was rapid during the white berry stage, gaining $\approx 60 \%$ of the final weight, and the levels of chlorophyll, phenolics, and PAL activity remained low and showed little change. One important feature of this development stage was the decline in chlorophyll content. Chlorophyll degradation likely was accompanied by that of the yellow carotenoids (Woodward, 1972), resulting in the white appearance of the fruit. Increase in the content of total soluble phenols per fruit, which was mostly due to accumulation of NT-flavonoids, commenced before and continued throughout the white berry stage. The particular flavonoid(s) that accumulated during this fruit expansion period was not determined. Ripe strawberry fruit contain considerable amounts of colorless flavonols and flavan monomers (Henning, 1981). The flavonol content in ripe strawberries is reportedly higher than that of anthocyanins (Pilando et al., 1985).

The persistent increase in the content of total soluble phenols, mostly flavonoids, throughout development of strawberry fruit is also found in pear (Billot et al., 1978). Continual production of flavonoids indicates that the phenylpropanoid and flavonoid pathways are intact and functional throughout fruit development. This may allow fruit tissues to respond rapidly to endogenous and external signals for new or enhanced synthesis of specific phenolic substances for particular developmental and defense requirements.

As fruit growth slowed and then ceased in the final development stage, two dramatic events occurred, both beginning at 23 DAA: PAL activity increased > 8-fold within 4 days, and anthocyanins accumulated at a remarkable rate. The average rate of accumulation was $3.8 \mu \mathrm{g} \cdot \mathrm{g}^{-1}$ fresh weight per hour (e.g., 23 to $30 \mathrm{DAA})$; $>75 \%$ of the final anthocyanin concentration was acquired within 2 days of the initiation of the rise. This rapid anthocyanin accumulation during ripening of strawberry fruit was observed in other cultivars (Given et al., 1988a; Woodward, 1972). During 23 to 27 DAA, 'Tillikum' fruit gained nearly $2.2 \mathrm{mg}$ of anthocyanins, or $<5 \mu \mathrm{mol}$, an amount easily accounted for by the potential substrate supply calculated from in vitro PAL activity (i.e., $52 \mu \mathrm{mol}$ cinnamic acid). Superabundant PAL activity in relation to actual phenolic synthesis is often found in plant cells and tissues (Margna, 1977). The final anthocyanin level in ripe 'Tillikum' fruit is similar to that of 'Totem' (Pilando et al., 1985), a highly colored cultivar, and higher than that in fruit of several other cultivars (Given et al., 1988a; Woodward, 1972).

The parallel rise in PAL activity and anthocyanin level was reported in two other strawberry cultivars (Given et al., 1988a; Hyodo, 1971), and fruit of several other species (Billet et al., 1978; Kataoka et al., 1983). Analogous changes in anthocyanin accumulation and PAL activity might reflect control of anthocyanin synthesis by PAL through the supply of cinnamic acid. Treatment of strawberry fruit with L- $\alpha$-aminooxy- $\beta$-phenylpropionic acid, an inhibitor of PAL, prevents fruit coloring (Given et al., 1988a). No anthocyanin accumulation was observed when PAL activity was undetectable in fruit of some grape (Kataoka et al., 1983) and apple (Kubo et al., 1988) cultivars. However, the apparent excess of PAL activity in 'Tillikum' fruit suggests that, although the enzyme is essential for anthocyanin synthesis, it might not be the rate-limiting enzyme. However, PAL may be a key factor controlling the channeling of phenylalanine into phenolic synthesis according to the physiological requirement of the fruit.

The rise in PAL activity observed in ripening strawberry fruit is, due to de novo enzyme synthesis (Given et al., 1988b). Transient transcription of PAL is observed in other systems (Kuhn et al., 1984) and could explain the sharp rise and fall back of the PAL activity observed during the final ripening of 'Tillikum' fruit. Involvement of a PAL inactivation system (PAL-IS) that causes an irreversible loss of enzyme activity in some plant tissues (Creasy, 1987), including skin of apple fruit (Blankenship and Unrath, 1988), has not been examined in strawberry.

The decline in PAL activity following the first peak in the green berry stage was slow compared with the precipitous fall in ripe fruit. This difference could be accounted for by several possibilities, including dissimilar systems regulating PAL activity or the consequence of separate genes encoding different PAL proteins in the two developmental stages (Liang et al., 1989). Although Given et al. (1988b) found that only a single isozyme of PAL is present in ripe 'Brighton' strawberry fruit, the isozyme pattern of PAL isolated from green fruit has not been determined.

Although flavonoids were synthesized throughout strawberry fruit development, anthocyanins only accumulated during ripening, concomitant with the second rise in PAL activity. This relationship suggests, as has been observed in other plant systems (Kuhn et al., 1984; Templeton and Lamb, 1988), that the phenylpropanoid pathway, as represented by PAL, is coordinated in ripening fruit with the activity of the enzymes involved in the synthesis of flavonoids, including anthocyanins. The last step in the biosynthesis of the major anthocyanin in strawberry, pelargonidin-3-glucoside, is glucosylation of the aglycone by UDP-glucose:flavonoid-3-O-glucosyltransferase (3-GT). The activity of 3-GT parallels that of PAL during fruit ripening (Given et al., 1988a). The failure of immature 'Tillikum' fruit to accumulate anthocyanins when they were competent to synthesize other flavonoids could have resulted from poor expression of 3GT or other enzymes closely associated with anthocyanin synthesis.

\section{Literature Cited}

Aoki, S., C. Awaki, K. Kaneko, and O. Katayama. 1970. L-phenylalanine ammonia-lyase activities in Japanese chestnut, strawberry, apple fruit, and bracken (in Japanese). Nippon Shokuhin Kogyo Gakkaish 19:507-511.

Aoki, S., C. Awaki, K. Kaneko, and O. Katayama. 1971. Occurrence of L-phenylalanine ammonia-lyase activity in peach fruit during growth. Agr. Biol. Chem. 35:784-787.

Billet, J., C. Hartmann, J. Macheix, and J. Rateau. 1978. Les composés phénoliques au tours de la crossance de la Poire Passe-Crassane. Physiol. Vég. 16:693-714.

Blankenship, S.M. and C.R. Unrath. 1988. PAL and ethylene content during maturation of Red and Golden Delicious apples. Photochemistry 27:1001-1003.

Bradford, M.M. 1976. A rapid and sensitive method for the quantitation of microgram quantities of protein utilizing the principle of protein-dye binding. Anal. Biochem. 72:248-254.

Creasy, L.L. 1987. The role of enzyme inactivation in the regu- 
lation of synthetic pathways: A case history. Physiol. Plant. 71:389392.

Foo, L.Y. and L.J. Porter. 1980. The photochemistry of proanthocyanidin polymers. Photochemistry 19:1747-1754.

Given, N. K., M.A. Venis, and D. Grierson. 1988a. Phenylalanine ammonia-lyase activity and anthocyanin synthesis in ripening strawberry fruit. J. Plant Physiol. 133:25-30.

Given, N. K., M.A. Venis, and D. Grierson. 1988b. Purification and properties of phenylalanine ammonia-lyase from strawberry fruit and its synthesis during ripening. J. Plant Physiol. 133:31-37.

Hahlbrock, K. and H. Griseback. 1979. Enzymic controls in the biosynthesis of lignin and flavonoids. Annu. Rev. Plant Physiol. 30:105130.

Henning, W. 1981. Flavonolglykoside der Erdbeeren (Fragaria $\times$ ananassa Duch.), Himbeeren (Rubus idaeus L.) und Brombeeren (Rubus fruticosus L.). 14. Phenolische Inhaltstoffe des Obstes. Z. Lebensm. Unters. Forsch. 173:180-187.

Hyodo, H. 1971. Phenylalanine ammonia-lyase in strawberry fruits. Plant Cell Physiol. 12:989-991.

Hyodo, H. and S. Asahara. 1973. Changes in phenylalanine ammonialyase activity in Satsuma mandarin fruit during ontogeny. Plant Cell Physiol. 14:823-828.

Ishikura, N., S. Hayashida, and K. Tazaki. 1984. Biosynthesis of gallic and ellagic acids with ${ }^{14} \mathrm{C}$-labeled compounds in Acer and Rhus leaves. Bet. Msg. Tokyo 97:355-367.

Jersch, S., C. Scherer, G. Huth, and E. Schlosser. 1989. Proanthocyanins as basis for quiescence of Botrytis cinera in immature strawberry fruits. Z. Pflkrankh. Pflschutz. 96:365-378.

Jones, D.H. 1984. Phenylalanine ammonia-lyase: Regulation of its induction, and its role in plant development.. Phytochemistry 23:13491359.

Kataoka, I., Y. Kubo,. A. Sugiura, and T. Tomana. 1983. Changes in L-phenylalanine ammonia-lyase activity and anthocyanin synthesis during berry ripening of three grape cultivars. J. Jpn. Soc. Hort. Sci. 52:273-279.

Kuhn, D., J. Chappell, A. Boudet, and K. Hahlbrick. 1984. Induction of phenylalanine ammonia-lyase and 4 coumarate: CoA ligase mRNAs in cultured plant cells by UV light or fungal elicitor. Proc. Natl. Acad. Sci. USA 8:1102-1106.

Kubo, Y., S. Taira, and S. Ishio. 1988. Color development of 4 apple cultivars grown in the southwest of Japan, with special reference to fruit bagging (in Japanese). J. Jpn. Soc. Hort. Sci. 57:191-199.

Liang, X., M. Dron, C.L. Cramer, R.A. Dixon, and C.J. Lamb. 1989. Differential regulation of phenylalanine ammonia-lyase genes during plant development and by environment cues. J. Biol. Chem. 264:14486-14492.

Macheix, J., A. Fleuriet, and J. Billet. 1990. Fruit phenolics. CRC, Boca Raton, Fla.

Mackinney, G. 1941. Absorption of light by chlorophyll solutions. J. Biol. Chem. 140:315-322.

Margna, U. 1977. Control at the level of substrate supply-an alternate in the regulation of accumulation in phenylpropanoid in plant cells. Photochemistry 16:419-426.

Melin, C., A. Moulet, J. Dupin, and C. Hartmann. 1977. Phenylalanine-ammoniaque lyase et composes phenoliques au tours de la maturation de la cerise. Photochemistry 16:75-78.

Peri, C. and C. Pompei. 1971. An assay of different phenolic fractions in wines. Amer. J. Enol. Vitic.22:55-58.

Pilando, L. B., R.E. Wrolstad, and D. A. Heatherbell. 1985. Influence of fruit composition, maturity and mold contamination on the color and appearance of strawberry wine. J. Food Sci. 50:1121-1125.

Sjulin, T.M. and B.H. Barritt. 1984. 'Tillikum' day-neutral strawberry. HortScience 19:452-453.

Slinkard, K. and V.L. Singleton. 1977. Total phenol analysis: automation and comparison with manual methods. Amer. J. Enol. Vitic. 28:49-55.

Spanos, G.A. and R.E. Wrolstad. 1990. Influence of processing and storage on the phenolic composition of Thompson Seedless grape juice. J. Agr. Food Chem. 38:1565-1571.

Spayd, S.E. and J.R. Morris. 1981. Physical and chemical characteristics of puree from once-over harvested strawberries. J. Amer. Soc. Hort. Sci. 106:105-109.

Templeton, D.D. and C.J. Lamb. 1988. Elicitors and defence gene activation. Plant Cell Environ. 11:395-401.

Woodward, J.R. 1972. Physical and chemical changes in developing strawberry fruits. J. Sci. Food. Agr. 23:465-473.

Zucker, M. 1965. Induction of phenylalanine deaminase by light and its relation to chlorogenic acid synthesis in potato tuber tissue. Plant Physiol. 40:779-784. 